\title{
Association between HDL-C levels and menopause: a meta-analysis
}

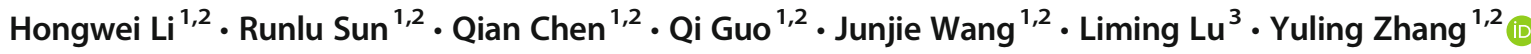

Received: 24 April 2020 / Accepted: 22 May 2020 / Published online: 18 June 2020

(C) The Author(s) 2020

\begin{abstract}
Purpose Menopause modifies women's lipid profiles. However, the fact that it is still unclear whether high-density lipoproteincholesterol (HDL-C) levels decrease in postmenopausal women necessitated a systematic review and meta-analysis.

Methods The PubMed, EMBASE, Cochrane Library, and Web of Science databases were searched and 498 articles published between 1987 and 2020 were retrieved. Studies reporting HDL-C, low-density lipoprotein-cholesterol (LDL-C), total cholesterol (TC), and triglyceride (TG) levels in both postmenopausal and premenopausal populations were included. The quality of the included studies was assessed using the Cross-Sectional/Prevalence Study Quality tool. The standard mean difference (SMD) and 95\% confidence interval (CI) were estimated using random effects models. A meta-regression analysis and subgroup analysis were performed to identify potential modifiers. Egger's test and funnel plots were constructed to evaluate publication biases. Results Lipid profiles from 18 cross-sectional studies and two cohort studies including 5652 postmenopausal women and 7825 premenopausal women were meta-analyzed. HDL-C levels were not significantly different between the postmenopausal and premenopausal women (SMD $=-0.053,95 \% \mathrm{CI}-0.171$ to $0.066, p=0.383$ ) and were not affected by country, publication year, study quality in the meta-regression analysis, or significant publication bias. Higher LDL-C, TC, and TG levels were detected in postmenopausal women than in premenopausal controls.

Conclusion Unlike increased LDL-C, TC, and TG levels, HDL-C levels in pre- and postmenopausal women were not different in this first meta-analysis of lipid profiles in premenopausal and postmenopausal women. Prospective studies with large populations examining HDL-C levels and functions in women with different menopausal statuses are essential in the future.
\end{abstract}

Trial registration number None.

Keywords High-density lipoprotein-cholesterol · Low-density lipoprotein-cholesterol $\cdot$ Triglycerides $\cdot$ Total cholesterol . Postmenopausal $\cdot$ Premenopausal $\cdot$ Cross-sectional

Electronic supplementary material The online version of this article (https://doi.org/10.1007/s42000-020-00216-8) contains supplementary material, which is available to authorized users.

Yuling Zhang

zzhangyuling@126.com

1 Cardiovascular Medicine Department, Sun Yat-sen Memorial Hospital, Sun Yat-sen University, No. 107, the West of Yanjiang Road, Guangzhou 510120, China

2 Guangdong Province Key Laboratory of Arrhythmia and Electrophysiology, Guangzhou 510120, China

3 Clinical Research Center, South China Research Center for Acupuncture and Moxibustion, Medical College of Acu-Moxi and Rehabilitation, Guangzhou University of Chinese Medicine, Guangzhou 510006, China

\section{Introduction}

The menopause transition is an inevitable physiological process that occurs in women and is characterized by biological changes such as reductions in estradiol levels and proatherogenic lipid profiles [1]. An increased risk of cardiovascular disease (CVD) has been observed during this phase of life. In particular, the risk doubles in women aged 4554 years [2], indicating harmful changes in the metabolic pattern between the pre- and postmenopausal periods. Highdensity lipoprotein cholesterol (HDL-C), a robust, independent, and traditional CVD risk factor, has exhibited inconsistent changes in a quantitative analysis of menopause-related changes. In addition to the dichotomous findings of either lower or higher HDL-C levels in postmenopausal women [3-5], recent cross-sectional studies did not identify a significant difference in HDL-C levels between pre- and postmenopausal women [6,7]. The long-established view 
acknowledges that HDL-C levels negatively correlate with the risk of CVD in the overall population [8]. However, accumulating evidence of a positive association between HDL-C levels and atherosclerosis-related diseases in postmenopausal women challenges the cardioprotective effect of high HDL-C levels $[9,10]$. An open-ended question is whether a high HDL-C level is a CVD risk marker after menopause. Elevated levels of low-density lipoprotein cholesterol (LDL$\mathrm{C})$, triglyceride (TG), and total cholesterol (TC) are related to CVD, with substantial interrelationships with HDL-C levels. An improved understanding of the changes in the levels of HDL-C and other lipids in response to menopause status will ensure a more reliable investment in strategies aiming to reduce CVD risk.

To our knowledge, we have performed the first metaanalysis to clarify whether HDL-C, LDL-C, TG, and TC levels are altered after the menopausal transition. The results of the meta-regression and subgroup analyses may explain the heterogeneity of changes in HDL-C levels during the menopause transition.

\section{Methods}

\section{Data sources and database searches}

We searched four public electronic databases, namely PubMed, Web of Science, EMBASE, and the Cochrane Library, for related articles. The following search terms were used: "Postmenopause" or "Postmenopausal period," and "Premenopause" or "Premenopausal period" in combination with "Lipoproteins, HDL" or "High-density Lipoproteins." The search was restricted to articles published in the English language that included human participants. Subsequent to our search, the articles included studies published from the beginning of 1987 to 2020 . All titles and abstracts yielded by the search were then screened for potential suitability. Those articles that appeared to be relevant were retrieved and examined in more detail. This search was supplemented by manual searches of the reference lists of all retrieved studies. We followed the Meta-analysis of Observational Studies in Epidemiology (MOOSE) guidelines to conduct and report the results of our meta-analyses [11].

\section{Inclusion and exclusion criteria}

We included comparative studies of postmenopausal and premenopausal women published in the English language that met the following criteria: (1) original articles that included, (2) both healthy pre- and postmenopausal women, and (3) simultaneously reported HDL-C, LDL-C, TC, and TG levels. Postmenopausal status was defined as more than 12 months of amenorrhea and premenopausal state was defined as regular menstrual cycles. Studies were excluded when (1) the pre- or postmenopausal women had lipid-altering diseases, including diabetes, coronary artery disease, malignancy, and thyroid functional abnormalities; (2) the pre- or postmenopausal women received hormone replacement therapy (HRT) or other medications affecting lipid metabolism; (3) the premenopausal women were pregnant; or (4) the women underwent menopause due to surgery or other unnatural causes. Studies were also excluded if they used only menopause status as a covariate or if they reported overlapping data or consisted of studies of a single case or reviews.

\section{Data extraction and methodological quality assessment}

Two authors (HWL and QC) independently extracted and summarized the data from the included studies. The papers were required to have indicated the total numbers of postmenopausal subjects and premenopausal subjects as well as the mean HDL-C level \pm standard deviation (SD) or the mean HDL-C level \pm standard error of mean (SE). When multiple publications reported the same or overlapping data, we included the most recent study with the largest sample size. The following data were extracted from each study: first author, year, country, sample sizes of the case and control groups, mean age, and the levels of TC, TG, LDL-C, and HDL-C, as well as their SD or SE. We extracted the baseline data from cohort studies if multiple time points were provided. The level of quality of all cross-sectional studies was evaluated using the Cross-Sectional/Prevalence Study Quality tool recommended by the Agency for Healthcare Research and Quality. An item received a score of " 1 " if the answer was "Yes" and a score of "0" if the answer was "No" or "Unclear" [12]. Studies with scores of 0-3 points were classified as "low quality," studies with a score of 4-7 points were classified as "moderate quality," and studies with a score of 8-11 points were classified as "high quality" [13]. Two reports were cohort studies [5, 14], and the same checklist was also used to assess their quality. One of the selected papers was a high-quality study and the remaining 19 papers were moderate quality. The majority of the included studies were considered to display an acceptable level of quality [see Table S1 in Additional file 1].

\section{Statistical analysis}

All statistical analyses were conducted using STATA software (version 15.0). A two-tailed $p$ value of $<0.05$ for any test or model was considered significant. All extracted data on TC, HDL-C, LDL-C, and TG levels were converted into mg/ dL. The pooled standardized mean difference (SMD) and its 95\% confidence interval (CI) were calculated for the metaanalysis of the lipid (HDL-C, LDL-C, TC, and TG) levels, which were included as continuous variables, in both cross- 
Fig. 1 Flow chart of the literature search and study selection process

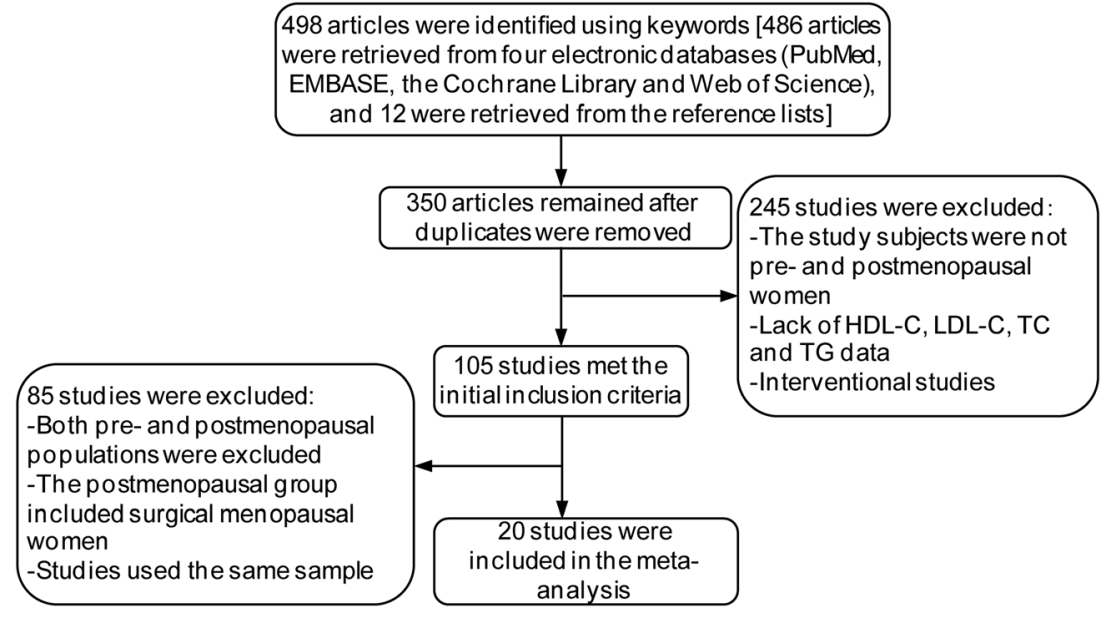

sectional studies and the baseline data from cohort studies, as mentioned in similar studies [15]. Heterogeneity was assessed using the $I^{2}$ statistic, which describes the percentage of variability in the effect estimates due to heterogeneity rather than chance. An approximated normality test (z-test) was used to analyze the aggregated results. Heterogeneity was considered significant when the probability value of the chi-square test was $<0.05$. As recommended by the Cochrane Handbookfor the Systematic Reviews of Interventions, an $I^{2}$ value $<40 \%$ suggests that "heterogeneity might not be important," whereas an $I^{2}$ value $>75 \%$ suggests "considerable heterogeneity." $\mathrm{We}$ pooled the data across trials using the fixed effects model (Mantel-Haenszel) if heterogeneity was absent or the random effects model if heterogeneity was present. A meta-regression was performed to explore the effects of publication year, sample size, country, and study quality on the effect estimates and identified the potential sources of the heterogeneity. Subgroup analyses were performed after stratification according to the publication year, sample size, and country to evaluate the association between HDL-C levels and menopausal status. Additionally, a sensitivity analysis was performed by repeating the meta-analysis after one study or a few studies were sequentially removed to assess whether a particular study significantly affected the pooled estimates. Potential publication bias was tested by creating a funnel plot that represented the standard error as the measure of the sample size and SMD as the measure of the pooled effect and Egger's test.

\section{Results}

\section{Search results}

The literature search yielded 498 related papers. Three hundred fifty studies remained after removing duplicates. Two hundred forty-five articles were then excluded because they did not include pre- and postmenopausal women as subjects; lacked HDL-C, LDL-C, TC, and TG data; and were interventional studies. One hundred and five studies met the initial inclusion criteria, of which 85 studies were excluded because of exclusion of both pre- and postmenopausal populations, inclusion of surgical menopausal women, and studies using the same populations. The remaining 20 original articles were included in the meta-analysis. The literature selection criteria and the reasons for exclusion are presented in Fig. 1. Eighteen of the 20 original articles were cross-sectional studies, and the remaining two articles were cohort studies.

\section{Study characteristics}

The characteristics of all 20 selected studies, which included 5652 postmenopausal women and 7825 premenopausal women, are summarized in Table 1 [4, 5, 7, 14, 16-31]. Asian participants from China, Korea, Japan, and India included 4979 postmenopausal women $(88.1 \%)$ and 7132 premenopausal controls $(91.1 \%)$, while non-Asian participants consisted of 673 postmenopausal women (11.9\%) and 693 premenopausal females $(8.9 \%)$. These eligible studies were conducted in ten countries, four of which were from the USA [4, 14, 23, 27], three articles were from Argentina [18, 24, 25], three articles were from Korea [19, 20, 28], two articles were from Japan [22, 31], two articles were from China $[5,23]$, and two articles were from Slovakia [7, 17]. The other four articles were from Brazil [21], India [16], Turkey [30], and the Netherlands [29].

\section{Association between menopause and HDL-C}

HDL-C levels in the postmenopausal and premenopausal women extracted from the 20 studies were synthesized. Due to heterogeneity, which was $>70 \%$, we conducted a metaanalysis of the 20 included studies using the random effects model to identify differences in HDL-C levels between postmenopausal women and premenopausal women (Fig. 2a). The 


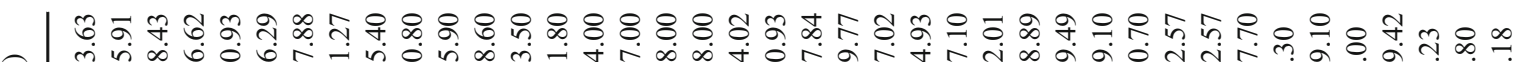
च $\quad$ च

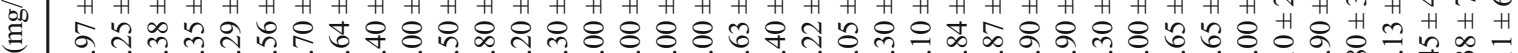

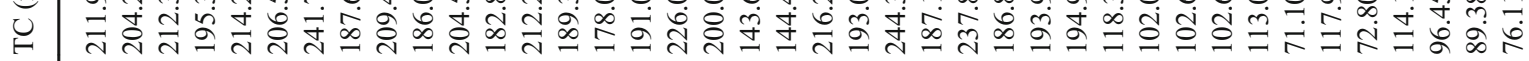

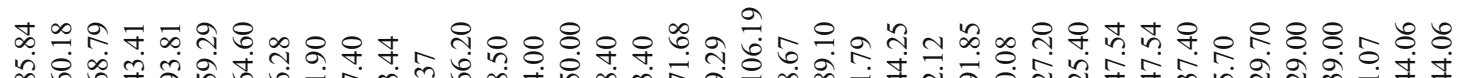

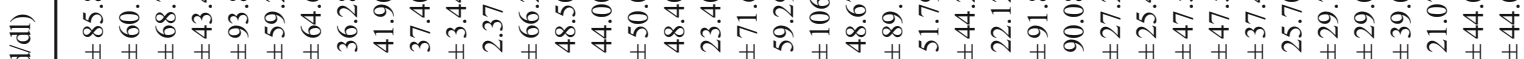

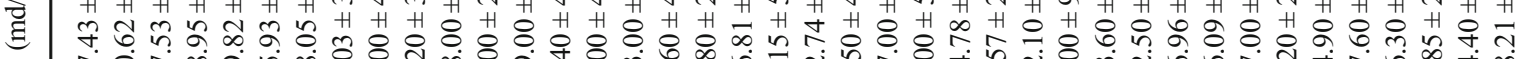
v $\quad$ ป

ิ

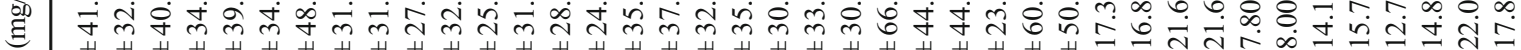
U

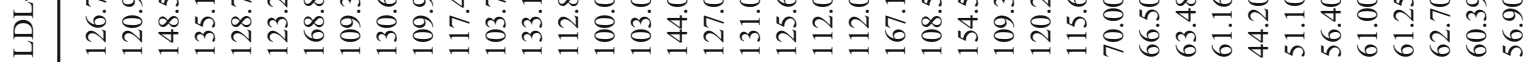

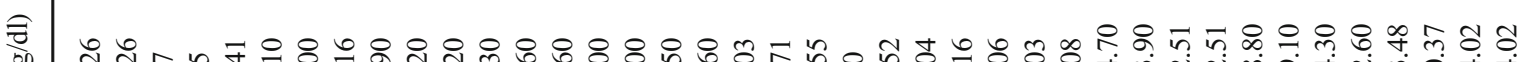
包 光

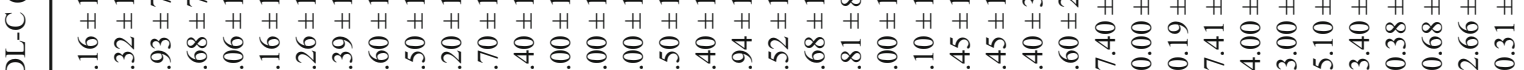

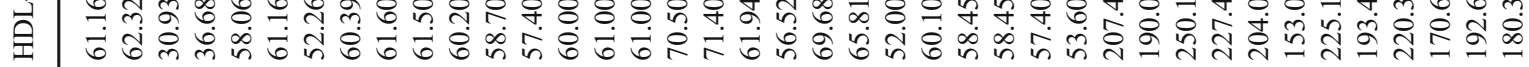

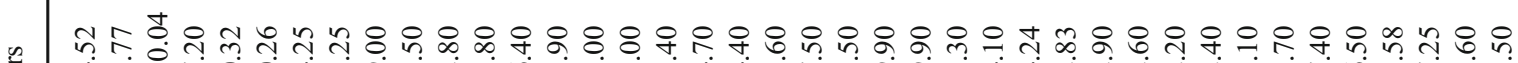

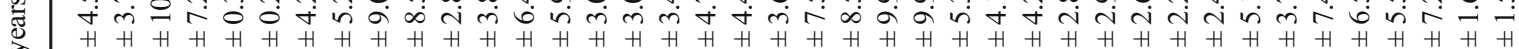

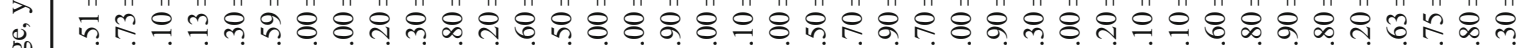

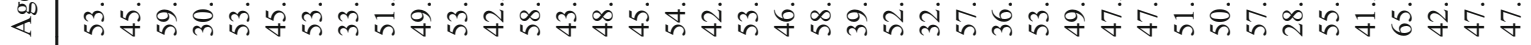

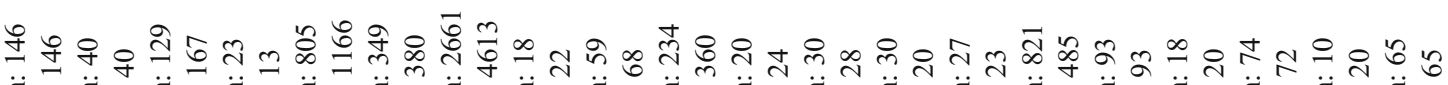

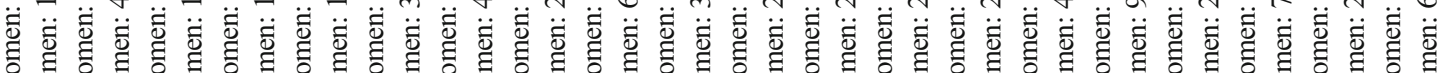

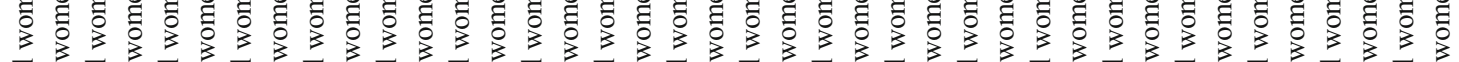

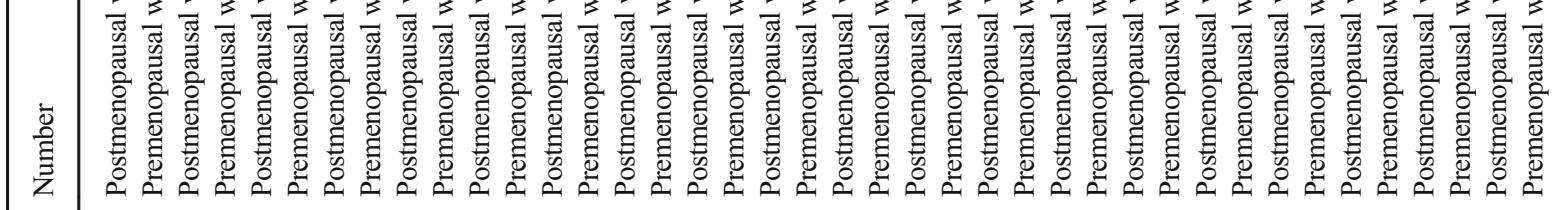

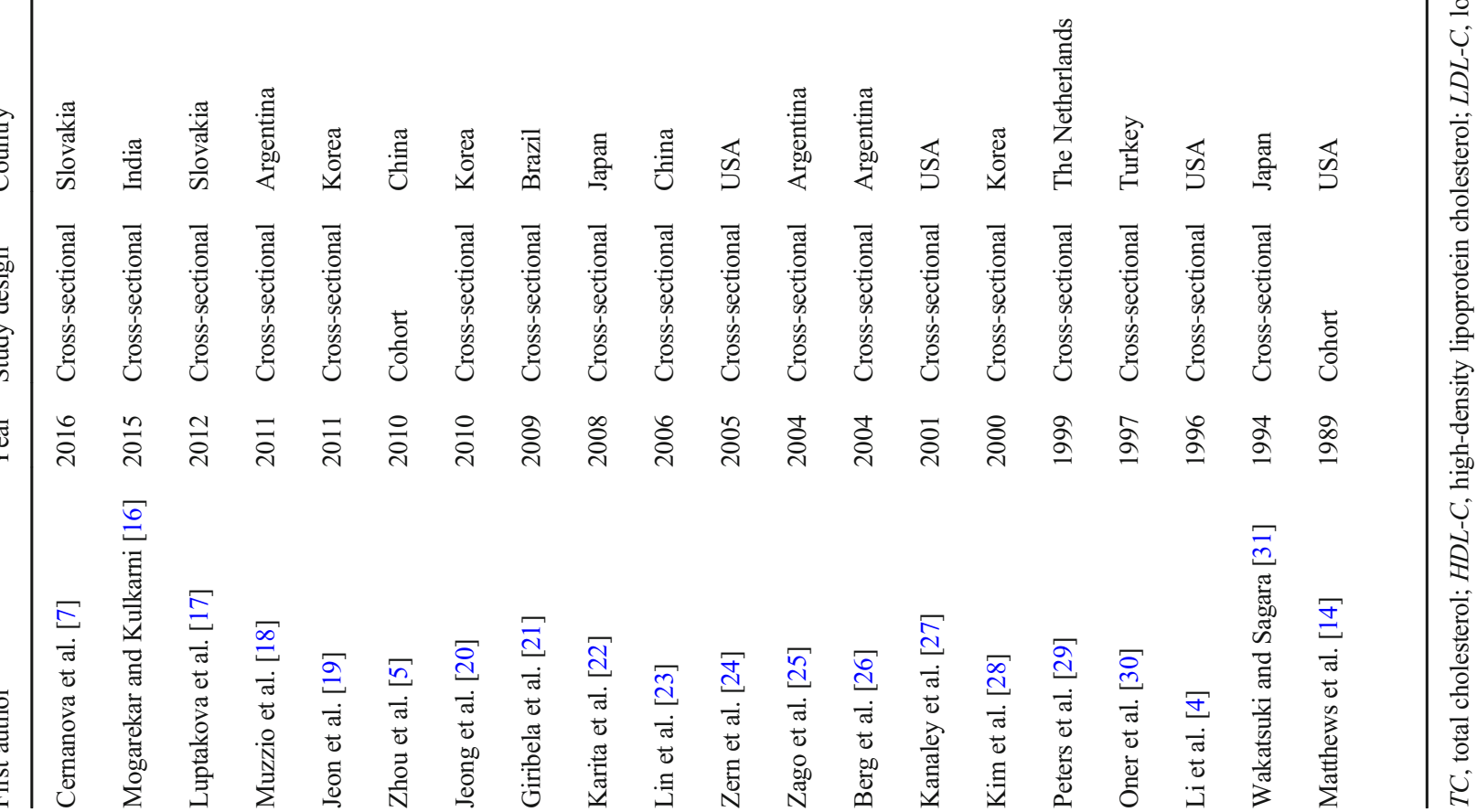


Fig. 2 Forest plots of the metaanalysis of HDL-C (a), LDL-C (b), TG (c), and TC (d) levels using random effects models. SMD standardized mean difference, CI confidence interval a

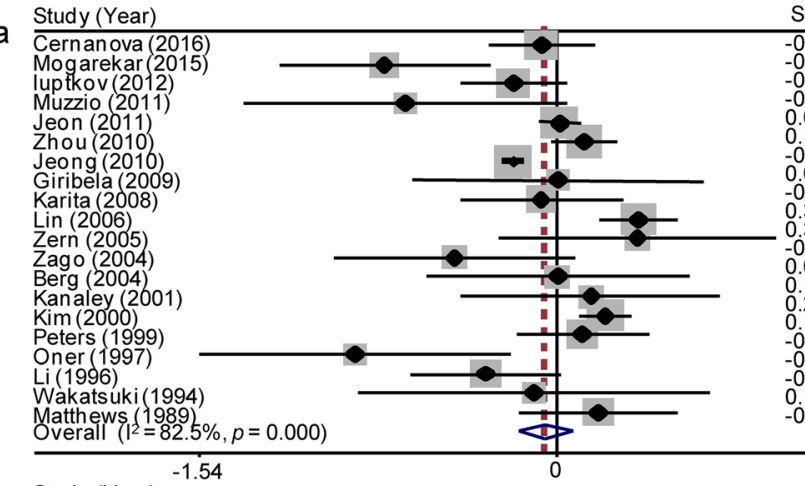

b

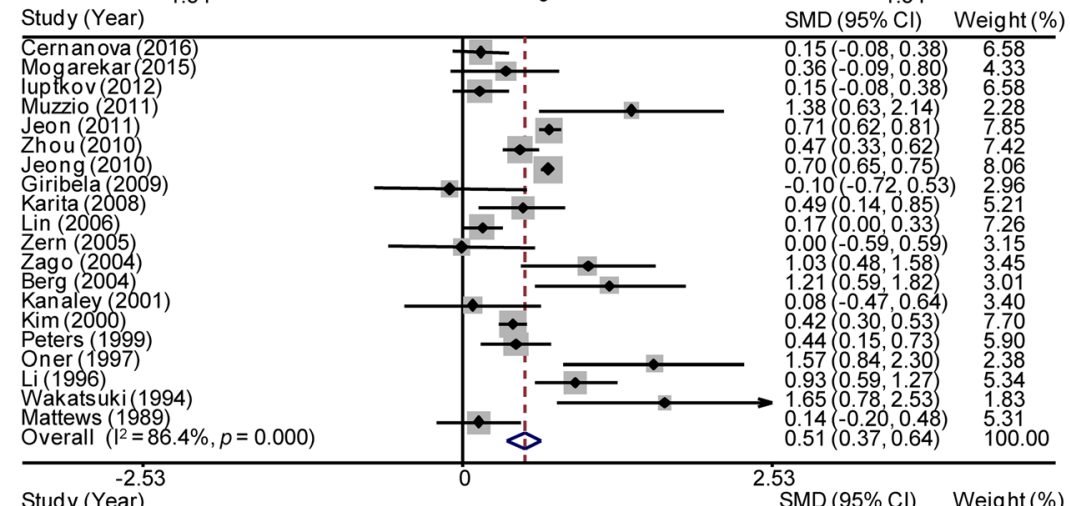

C

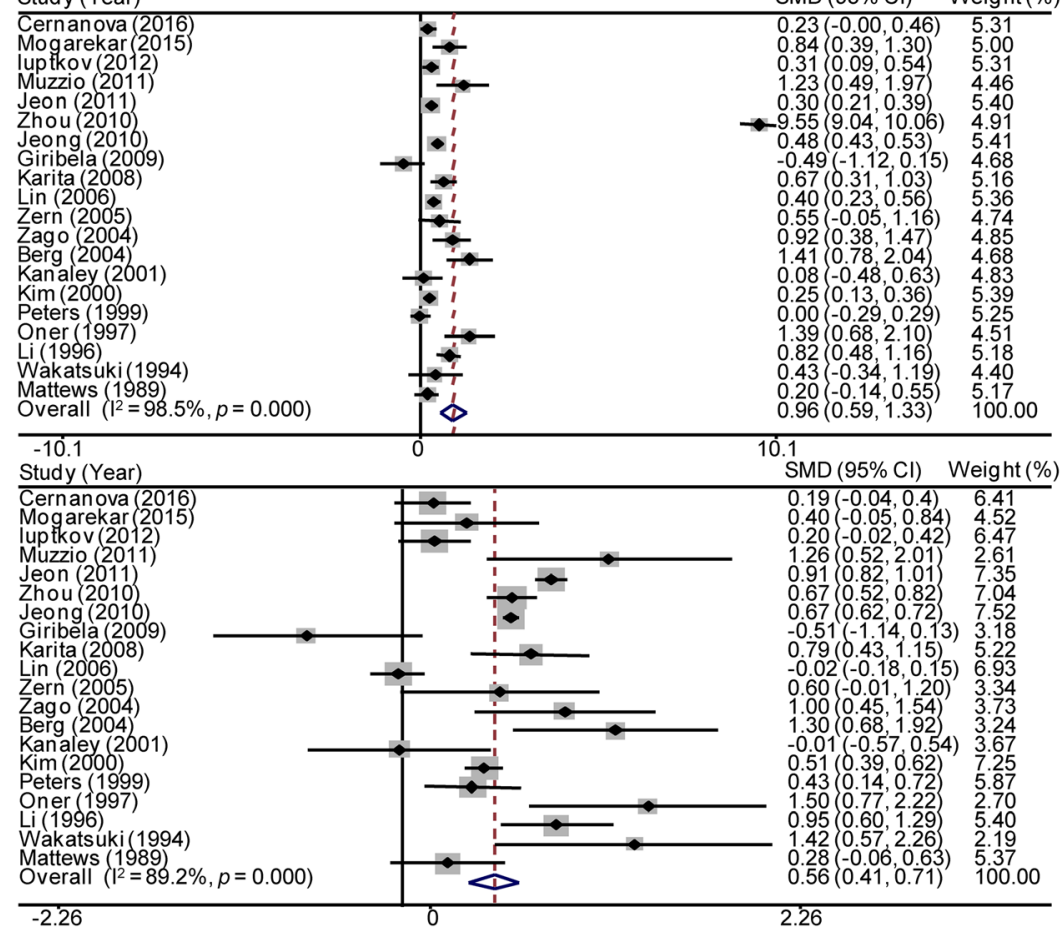

HDL-C levels were not significantly different between postmenopausal and premenopausal women $(\mathrm{SMD}=-0.053$, $95 \% \mathrm{CI}-0.171$ to $0.066, p=0.383$ ). Significant heterogeneity was observed among the available studies $(p<0.001$ and $I^{2}=82.5 \%$ for the heterogeneity test). A meta-regression analysis was conducted to identify potential moderators of this heterogeneity, but associations between HDL-C levels and covariates, including the publication year, sample size, and country, were not observed (Table 2). We performed a subgroup analysis after stratification by publication year, sample size, and country (Table 3 ). In the subgroups with a sample size $\leq 500$ and non-Asian countries, the $I^{2}$ value was significantly reduced by more than $20 \%$. In the sensitivity analysis, no studies which were sequentially omitted affected the 
Table 2 The meta-regression analysis of HDL-C, LDL-C, TG, and TC levels

\begin{tabular}{|c|c|c|c|c|}
\hline & \multirow[b]{2}{*}{$B$} & \multicolumn{2}{|l|}{$95 \% \mathrm{CI}$} & \multirow[b]{2}{*}{$p$} \\
\hline & & Lower CI limit & Upper CI limit & \\
\hline \multicolumn{5}{|l|}{ HDL-C } \\
\hline Publication year & 0.094 & -0.123 & 0.312 & 0.360 \\
\hline Sample size & 0.000 & -0.000 & 0.000 & 0.823 \\
\hline Country & 0.096 & -0.118 & 0.311 & 0.344 \\
\hline Study quality & 0.064 & -0.045 & 0.173 & 0.235 \\
\hline \multicolumn{5}{|l|}{ LDL-C } \\
\hline Publication year & -0.014 & -0.045 & 0.017 & 0.350 \\
\hline Sample size & 0.000 & -0.000 & 0.000 & 0.734 \\
\hline Country & -0.042 & -0.120 & 0.036 & 0.276 \\
\hline Study quality & -0.067 & -0.244 & 0.109 & 0.433 \\
\hline \multicolumn{5}{|l|}{$\mathrm{TG}$} \\
\hline Publication year & 0.044 & -0.094 & 0.183 & 0.508 \\
\hline Sample size & 0.000 & -0.001 & 0.001 & 0.951 \\
\hline Country & -0.187 & -0.519 & 0.145 & 0.252 \\
\hline Study quality & 1.090 & 0.454 & 1.726 & $0.002 *$ \\
\hline \multicolumn{5}{|l|}{$\mathrm{TC}$} \\
\hline Publication year & -0.013 & -0.045 & 0.019 & 0.395 \\
\hline Sample size & 0.000 & -0.000 & 0.000 & 0.826 \\
\hline Country & -0.024 & -0.106 & 0.059 & 0.558 \\
\hline Study quality & -0.036 & -0.223 & 0.152 & 0.693 \\
\hline
\end{tabular}

The correlation coefficients of publication year, sample size, country, and study quality with $95 \%$ confidence intervals (CI) for the effect size of HDL-C, LDL-C, TG, and TC of this meta-analysis are presented and considered significant to be heterogeneity sources with $p<0.05(*)$

association between the menopause status and HDL-C levels (Fig. 3a). A funnel plot was generated and Egger's test was performed to assess the publication bias of the studies included in this meta-analysis. The shapes of the funnel plots did not reveal any obvious asymmetry and Egger's test revealed $\beta=$ 0.390 and $p=0.616$. Both the appearance of the funnel plot and Egger's test results confirmed a lack of significant publication bias (Fig. 3e).

\section{Association between menopause and the levels of other lipid components}

Higher LDL-C $(\mathrm{SMD}=0.507,95 \% \mathrm{CI}=0.373$ to 0.642 , $p<0.001$ ) (Fig. $2 b$ ), $\mathrm{TG}$ ( $\mathrm{SMD}=0.958,95 \% \mathrm{CI}=0.587$ to $1.330, p<0.001)$ (Fig. 2c), and TC levels (SMD $=0.563,95 \%$ $\mathrm{CI}=0.415$ to $0.711, p<0.001$ ) (Fig. $2 \mathrm{~d}$ ) were detected in postmenopausal women than in premenopausal women. No associations between the modifiers publication year, sample size, country, and LDL-C, or TC levels were identified (Table 2) in meta-regression analyses. However, TG levels tended to be affected by study quality (Table 2). The subgroups of a sample size of $\leq 500$, year $<2008$, and non-Asian countries presented a greater than $20 \%$ decrease in the $I^{2}$ value. For TC and LDL-C levels, no subgroup showed a significant reduction in the $I^{2}$ value (Table 3 ). The sensitivity analysis revealed a decrease in the random effects estimates after omitting the study conducted by Zhou et al., [5] indicating that this study was a potential source of heterogeneity (Fig. 3c). The funnel plot of the metaanalysis of TG levels showed apparent asymmetry (Fig. 3g), although Egger's test did not reveal significant publication bias ( $\beta=2.779, p=0.281$ ). After removing this study, no association was observed between TG levels and study quality in the metaregression analysis $(\beta=-0.084, p=0.467)$, but the synthesized higher TG levels observed in postmenopausal women compared with premenopausal controls were not affected $(\mathrm{SMD}=0.433$, $95 \%$ CI 0.319 to $-0.548, p<0.001)$. In the sensitivity analysis, no studies which were sequentially omitted affected the association between the menopause status and LDL-C and TC levels (LDL-C: Fig. 3b; TC: Fig. 3d). Both the appearance of the funnel plot (LDL-C: Fig. 3f; TC: Fig. 3h) and Egger's test results (LDL-C: $\beta=-1.015, p=0.242$; TC: $\beta=-0.720, p=0.465$ ) showed no significant publication bias in the meta-analysis of LDL-C and TC levels in postmenopausal and premenopausal women.

\section{Discussion}

To the best of our knowledge, this is the first meta-analysis conducted to identify an association between the menopause status and blood lipid profiles. Based on the results of our study, HDL-C levels in postmenopausal women were not significantly different from those in premenopausal women. This finding is consistent with some large population studies [5, 19], which indicated that menopause did not affect HDL-C levels. In the subgroup analysis, sample size $\leq 500$ and nonAsian countries affected the heterogeneity of this meta-analysis. Although the value was not significant, a small sample size potentially affected the association between HDL-C levels and menopause status (Table 3). The difference in HDL-C levels between pre- and postmenopausal women potentially originates from the analysis of cross-sectional data. Wang et al. [32] identified significantly lower HDL-C levels in postmenopausal women than in premenopausal women in longitudinal studies but not in cross-sectional studies. Due to insufficient evidence of HDL-C changes during the menopause transition, more large population-based prospective studies are crucial to determine the association between HDL-C levels and the menopause transition.

Unlike HDL-C levels, LDL-C, TG, and TC levels were significantly increased in postmenopausal women compared with premenopausal controls. A recent large population-based study also observed significantly higher LDL-C and TC levels in postmenopausal women than in premenopausal women, 
Table 3 The subgroup analyses of HDL-C, LDL-C, TG, and TC levels

\begin{tabular}{|c|c|c|c|c|c|}
\hline & $N$ & $\operatorname{SMD}(95 \% \mathrm{CI})(\mathrm{mg} / \mathrm{dl})$ & $p$ & $I^{2}(\%)$ & Weight (\%) \\
\hline \multicolumn{6}{|l|}{ HDL-C } \\
\hline Total & 20 & $-0.053(-0.171,0.066)$ & 0.383 & 82.5 & 100.00 \\
\hline \multicolumn{6}{|l|}{ Sample size } \\
\hline$>500$ & 5 & $0.089(-0.109,0.286)$ & 0.379 & 95.0 & 40.73 \\
\hline$\leq 500$ & 15 & $-0.147(-0.296,0.001)$ & 0.052 & 47.1 & 59.27 \\
\hline \multicolumn{6}{|l|}{ Year } \\
\hline$\geq 2008$ & 9 & $-0.119(-0.247,0.010)$ & 0.070 & 77.8 & 51.89 \\
\hline$<2008$ & 11 & $0.036(-0.137,0.210)$ & 0.681 & 65.4 & 48.11 \\
\hline \multicolumn{6}{|l|}{ Country } \\
\hline Asia & 10 & $-0.024(-0.190,0.142)$ & 0.775 & 90.7 & 58.91 \\
\hline Not Asia & 10 & $-0.105(-0.242,0.032)$ & 0.134 & 20.9 & 41.09 \\
\hline \multicolumn{6}{|l|}{ LDL-C } \\
\hline Total & 20 & $0.507(0.373,0.642)$ & $<0.001$ & 86.4 & 100.00 \\
\hline \multicolumn{6}{|l|}{ Sample size } \\
\hline$>500$ & 5 & $0.506(0.332,0.680)$ & $<0.001$ & 93.3 & 38.29 \\
\hline$\leq 500$ & 15 & $0.553(0.323,0.783)$ & $<0.001$ & 77.5 & 61.71 \\
\hline \multicolumn{6}{|l|}{ Year } \\
\hline$\geq 2008$ & 9 & $0.467(0.304,0.631)$ & $<0.001$ & 86.6 & 51.27 \\
\hline$<2008$ & 11 & $0.584(0.353,0.815)$ & $<0.001$ & 80.6 & 48.73 \\
\hline \multicolumn{6}{|l|}{ Country } \\
\hline Asia & 10 & $0.529(0.373,0.686)$ & $<0.001$ & 88.9 & 57.36 \\
\hline Non-Asian country & 10 & $0.490(0.215,0.766)$ & $<0.001$ & 78.4 & 42.64 \\
\hline \multicolumn{6}{|l|}{ TG } \\
\hline Total & 20 & $0.958(0.587,1.330)$ & $<0.001$ & 98.5 & 100.00 \\
\hline \multicolumn{6}{|l|}{ Sample size } \\
\hline$>500$ & 5 & $2.094(1.261,2.926)$ & $<0.001$ & 99.7 & 26.47 \\
\hline$\leq 500$ & 15 & $0.529(0.308,0.749)$ & $<0.001$ & 75.7 & 73.53 \\
\hline \multicolumn{6}{|l|}{ Year } \\
\hline$\geq 2008$ & 9 & $1.436(0.727,2.146)$ & $<0.001$ & 99.4 & 45.64 \\
\hline$<2008$ & 11 & $0.500(0.291,0.710)$ & $<0.001$ & 76.2 & 54.36 \\
\hline \multicolumn{6}{|l|}{ Country } \\
\hline Asia & 10 & $0.467(0.190,0.744)$ & $<0.001$ & 99.3 & 49.29 \\
\hline Non-Asian country & 10 & $1.416(0.842,1.991)$ & 0.001 & 79.0 & 50.71 \\
\hline \multicolumn{6}{|l|}{$\mathrm{TC}$} \\
\hline Total & 20 & $0.563(0.415,0.711)$ & $<0.001$ & 89.2 & 100.00 \\
\hline \multicolumn{6}{|l|}{ Sample size } \\
\hline$>500$ & 5 & $0.555(0.331,0.779)$ & $<0.001$ & 96.0 & 36.09 \\
\hline$\leq 500$ & 15 & $0.592(0.363,0.822)$ & $<0.001$ & 77.5 & 63.91 \\
\hline \multicolumn{6}{|l|}{ Year } \\
\hline$\geq 2008$ & 9 & $0.535(0.352,0.718)$ & $<0.001$ & 89.5 & 50.32 \\
\hline$<2008$ & 11 & $0.634(0.374,0.894)$ & $<0.001$ & 85.2 & 49.68 \\
\hline \multicolumn{6}{|l|}{ Country } \\
\hline Asia & 10 & $0.610(0.427,0.793)$ & $<0.001$ & 92.1 & 56.08 \\
\hline Non-Asian country & 10 & $0.511(0.229,0.793)$ & $<0.001$ & 79.6 & 43.92 \\
\hline
\end{tabular}

$S M D$, standardized mean difference; $C I$, confidential interval while HDL-C levels were not different between postmenopausal and premenopausal women [33]. Menopause-induced proatherogenic lipid profile changes may mainly affect LDLC, TG, and TC levels. 

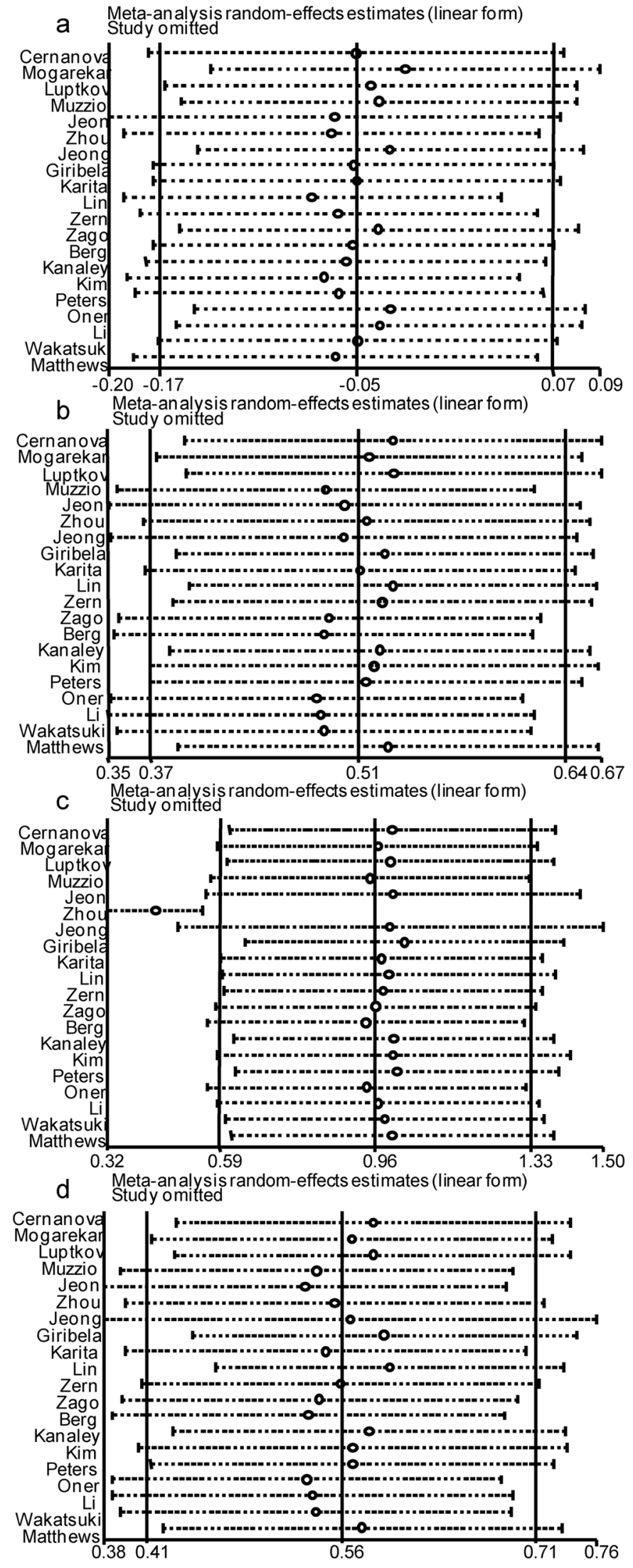

e
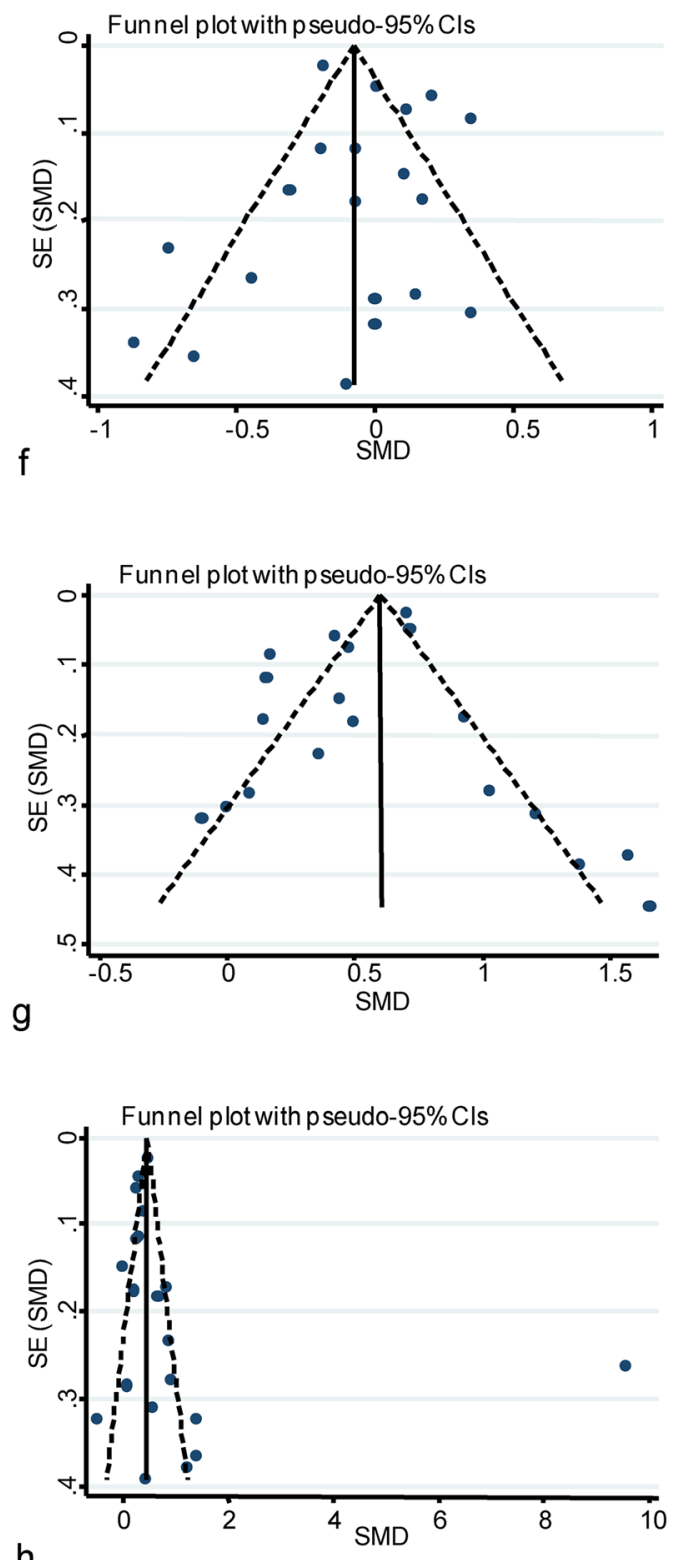

$\mathrm{h}$

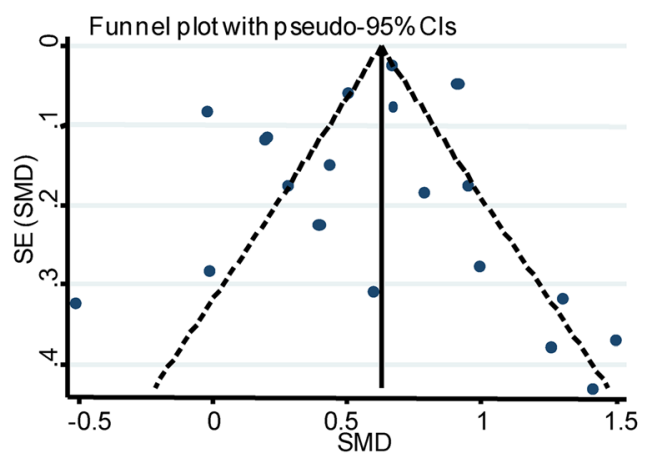

Fig. 3 Sensitivity analysis and funnel plot of the meta-analysis of HDL-C, LDL-C, TG, and TC levels. Sensitivity analysis (a, b, c, d) and funnel plots (e, $\mathbf{f}, \mathbf{g}, \mathbf{h}$ ) were performed to examine the heterogeneity of this meta-analysis. SMD standardized mean difference, CI confidence interval 
The study quality of the included articles was not associated with HDL-C, LDL-C, TG, and TC in metaregression analyses (Table 2). We strictly followed the inclusion and exclusion criteria to include relevant studies in our meta-analysis. No significant source of heterogeneity was identified and no low-quality studies were included. The sensitivity analysis identified the study conducted by Zhou et al. [5] as a source of heterogeneity in the pooled analysis of TG levels (Fig. 3c), but its omission did not affect the conclusion of increased TG levels in postmenopausal women compared with premenopausal women.

Besides quantitative HDL-C levels, novel metrics for evaluating HDL quality strongly pointed to these levels being independent cardiovascular risk factors. Compared with HDL levels of premenopausal women, less oxidative resistance of HDL among postmenopausal women was demonstrated, together with an impaired ability to inhibit LDL oxidation [25]. On the other hand, large HDL particle concentrations and HDL-mediated cholesterol efflux capacity has been reported to be increased in the early menopausal transition [34]. These novel HDL metrics may explain the pathophysiological changes in late perimenopausal and postmenopausal women, which needs to be confirmed by acquiring additional evidence and performing further studies.

Estrogen, which is the most important female sex hormone, displays decreased levels in postmenopausal women. Hormone replacement therapy and estrogen receptor modulators have been reported to improve lipoprotein levels in postmenopausal populations [35, 36]. Additional studies are needed to explore the effects of sex hormones on postmenopausal lipid functions.

\section{Strengths and limitations}

Our study is, as far as we know, the first meta-analysis to compare the association of HDL-C levels between healthy postmenopausal and premenopausal women, which increases the knowledge base regarding the potential association between HDL-C levels and the menopause status. One of the limitations of this study is that most of the participants came from Asia because of large sample size-based Korean and Japanese studies; thus, more clinical data derived from further studies from Western countries are required to better represent global populations. The definition of menopause status is based on the time from the last bleeding pattern, regardless of sex hormone levels. Additionally, although some studies have used FSH levels as a criterion to confirm menopause status, consensus on the cut-off values for postmenopausal FSH levels has not as yet been established $[22,28]$. Another limitation is the small number of included cohort studies and sample sizes; therefore, further prospective studies would help to improve the inferences of causality.

\section{Conclusions}

Based on the evidence of this meta-analysis, HDL-C levels are not associated with menopause status. Higher LDL-C, TG, and TC levels are observed in postmenopausal women than in premenopausal women. Prospective studies with large sample sizes examining HDL-C levels and HDL functions in women with different menopause statuses are needed.

Authors' contributions HWL mainly performed the search for the relevant articles, extracted the data, performed the statistical analyses, and wrote the paper. RLS participated in drafting the paper and settling any conflicts regarding article inclusion. QC helped search for articles, extract the data from the included articles, and evaluate the quality of the articles by assessing the methodological quality using the Cross-Sectional/ Prevalence Study Quality tool. QG and JJW generated the figures and tables. LML provided guidance for the statistical analyses. YLZ was the chief investigator who directed this study. All authors read and approved the final manuscript.

Funding information The authors disclose the receipt of the following financial support for the research, authorship, and/or publication of this article: this work was supported by the Natural Science Foundation of China (No. 81970388), the Key S\&T Special Project of Guangzhou (201704020044), and the Yat-sen Scientific Research Launching Project (YXQH201804).

Data availability Not applicable.

\section{Compliance with ethical standards}

Conflict of interest The authors declare that they have no conflict of interest.

Ethics approval Not applicable.

Consent to participate Not applicable.

Consent for publication Not applicable.

Code availability Not applicable

Open Access This article is licensed under a Creative Commons Attribution 4.0 International License, which permits use, sharing, adaptation, distribution and reproduction in any medium or format, as long as you give appropriate credit to the original author(s) and the source, provide a link to the Creative Commons licence, and indicate if changes were made. The images or other third party material in this article are included in the article's Creative Commons licence, unless indicated otherwise in a credit line to the material. If material is not included in the article's Creative Commons licence and your intended use is not permitted by statutory regulation or exceeds the permitted use, you will need to obtain permission directly from the copyright holder. To view a copy of this licence, visit http://creativecommons.org/licenses/by/4.0/. 


\section{References}

1. El Khoudary SR (2017) Gaps, limitations and new insights on endogenous estrogen and follicle stimulating hormone as related to risk of cardiovascular disease in women traversing the menopause: a narrative review. Maturitas 104:44-53. https://doi.org/10. 1016/j.maturitas.2017.08.003

2. Gordon T, Kannel WB, Hjortland MC, McNamara PM (1978) Menopause and coronary heart disease. The Framingham Study. Ann Intern Med 89(2):157-161

3. Matthan NR, Jalbert SM, Lamon-Fava S, Dolnikowski GG, Welty FK, Barrett HR, Schaefer EJ, Lichtenstein AH (2005) TRL, IDL, and LDL apolipoprotein B-100 and HDL apolipoprotein A-I kinetics as a function of age and menopausal status. Arterioscler Thromb Vasc Biol 25(8):1691-1696. https://doi.org/10.1161/01.ATV. 0000172629.12846.b8

4. Li Z, McNamara JR, Fruchart JC, Luc G, Bard JM, Ordovas JM, Wilson PW, Schaefer EJ (1996) Effects of gender and menopausal status on plasma lipoprotein subspecies and particle sizes. J Lipid Res 37(9):1886-1896

5. Zhou JL, Lin SQ, Shen Y, Chen Y, Zhang Y, Chen FL (2010) Serum lipid profile changes during the menopausal transition in Chinese women: a community-based cohort study. Menopause 17(5):997-1003. https://doi.org/10.1097/gme.0b013e3181dbdc30

6. Cho EJ, Min YJ, Oh MS, Kwon JE, Kim JE, Lee WS, Lee KJ, Kim SW, Kim TH, Kim MA, Kim CJ, Ryu WS (2011) Effects of the transition from premenopause to postmenopause on lipids and lipoproteins: quantification and related parameters. Korean J Intern Med 26(1):47-53. https://doi.org/10.3904/kjim.2011.26.1.47

7. Cernanova V, Dankova Z, Luptakova L, Cvicelova M, Sivakova D (2016) The association of Asn453Ser polymorphism in CYP1B1 gene with selected somatic and biochemical variables in Slovak women of different menopause status. Menopause 23(5):577-583. https://doi.org/10.1097/GME.0000000000000565

8. Castelli WP, Garrison RJ, Wilson PW, Abbott RD, Kalousdian S, Kannel WB (1986) Incidence of coronary heart disease and lipoprotein cholesterol levels. The Framingham Study. Jama 256(20): 2835-2838

9. Fan AZ, Dwyer JH (2007) Sex differences in the relation of HDL cholesterol to progression of carotid intima-media thickness: the Los Angeles atherosclerosis study. Atherosclerosis 195(1):e191e196. https://doi.org/10.1016/j.atherosclerosis.2007.03.045

10. El Khoudary SR, Wang L, Brooks MM, Thurston RC, Derby CA, Matthews KA (2016) Increase HDL-C level over the menopausal transition is associated with greater atherosclerotic progression. $\mathrm{J}$ Clin Lipidol 10(4):962-969. https://doi.org/10.1016/j.jacl.2016.04. 008

11. Stroup DF, Berlin JA, Morton SC, Olkin I, Williamson GD, Rennie D, Moher D, Becker BJ, Sipe TA, Thacker SB (2000) Metaanalysis of observational studies in epidemiology: a proposal for reporting. Meta-analysis Of Observational Studies in Epidemiology (MOOSE) group. Jama 283(15):2008-2012

12. AHRQ (2008) Comparative effectiveness reviews. AHRQ, Rockville

13. Guan XL, Wang H, Huang HS, Meng L (2015) Prevalence of dysphagia in multiple sclerosis: a systematic review and meta-analysis. Neurol Sci 36(5):671-681. https://doi.org/10.1007/s10072015-2067-7

14. Matthews KA, Meilahn E, Kuller LH, Kelsey SF, Caggiula AW, Wing RR (1989) Menopause and risk factors for coronary heart disease. N Engl J Med 321(10):641-646. https://doi.org/10.1056/ NEJM198909073211004

15. Solmi M, Veronese N, Favaro A, Santonastaso P, Manzato E, Sergi G, Correll CU (2015) Inflammatory cytokines and anorexia nervosa: a meta-analysis of cross-sectional and longitudinal studies.
Psychoneuroendocrinology 51:237-252. https://doi.org/10.1016/j. psyneuen.2014.09.031

16. Mogarekar MR, Kulkarni SK (2015) Small dense low density lipoprotein cholesterol, paraoxonase 1 and lipid profile in postmenopausal women: quality or quantity? Arch Med Res 46(7):534-538. https://doi.org/10.1016/j.arcmed.2015.08.007

17. Luptakova L, Sivakova D, Sramekova D, Cvicelova M (2012) The association of cytochrome P450 1B1 Leu432Val polymorphism with biological markers of health and menopausal symptoms in Slovak midlife women. Menopause 19(2):216-224. https://doi. org/10.1097/gme.0b013e3182281b54

18. Muzzio ML, Miksztowicz V, Repetto EM, Brites F, Berg G, Schreier L (2012) Increased MMP-2 in healthy postmenopausal women. Ann Clin Biochem 49(Pt 1):75-79. https://doi.org/10. 1258/acb.2011.011041

19. Jeon YK, Lee JG, Kim SS, Kim BH, Kim SJ, Kim YK, Kim IJ (2011) Association between bone mineral density and metabolic syndrome in pre- and postmenopausal women. Endocr J 58(2): 87-93

20. Jeong IK, Cho SW, Kim SW, Choi HJ, Park KS, Kim SY, Lee HK, Cho SH, Oh BH, Shin CS (2010) Lipid profiles and bone mineral density in pre- and postmenopausal women in Korea. Calcif Tissue Int 87(6):507-512. https://doi.org/10.1007/s00223-010-9427-3

21. Giribela AH, Melo NR, Latrilha MC, Baracat EC, Maranhao RC (2009) HDL concentration, lipid transfer to HDL, and HDL size in normolipidemic nonobese menopausal women. Int J Gynaecol Obstet 104(2):117-120. https://doi.org/10.1016/j.ijgo.2008.10.001

22. Karita K, Yamanouchi Y, Takano T, Oku J, Kisaki T, Yano E (2008) Associations of blood selenium and serum lipid levels in Japanese premenopausal and postmenopausal women. Menopause 15(1):119-124. https://doi.org/10.1097/gme. 0b013e31806bf32c

23. Lin WY, Yang WS, Lee LT, Chen CY, Liu CS, Lin CC, Huang KC (2006) Insulin resistance, obesity, and metabolic syndrome among non-diabetic pre- and post-menopausal women in North Taiwan. Int J Obes 30(6):912-917. https://doi.org/10.1038/sj.ijo.0803240

24. Zern TL, Wood RJ, Greene C, West KL, Liu Y, Aggarwal D, Shachter NS, Fernandez ML (2005) Grape polyphenols exert a cardioprotective effect in pre- and postmenopausal women by lowering plasma lipids and reducing oxidative stress. J Nutr 135(8): 1911-1917. https://doi.org/10.1093/jn/135.8.1911

25. Zago V, Sanguinetti S, Brites F, Berg G, Verona J, Basilio F, Wikinski R, Schreier L (2004) Impaired high density lipoprotein antioxidant activity in healthy postmenopausal women. Atherosclerosis 177(1):203-210. https://doi.org/10.1016/j. atherosclerosis.2004.07.011

26. Berg G, Mesch V, Boero L, Sayegh F, Prada M, Royer M, Muzzio ML, Schreier L, Siseles N, Benencia H (2004) Lipid and lipoprotein profile in menopausal transition. Effects of hormones, age and fat distribution. Horm Metab Res 36(4):215-220. https://doi.org/10. 1055/s-2004-814450

27. Kanaley JA, Sames C, Swisher L, Swick AG, Ploutz-Snyder LL, Steppan CM, Sagendorf KS, Feiglin D, Jaynes EB, Meyer RA, Weinstock RS (2001) Abdominal fat distribution in pre- and postmenopausal women: the impact of physical activity, age, and menopausal status. Metabolism 50(8):976-982. https://doi.org/10.1053/ meta.2001.24931

28. Kim CJ, Kim TH, Ryu WS, Ryoo UH (2000) Influence of menopause on high density lipoprotein-cholesterol and lipids. J Korean Med Sci 15(4):380-386. https://doi.org/10.3346/jkms.2000.15.4. 380

29. Peters HW, Westendorp IC, Hak AE, Grobbee DE, Stehouwer CD, Hofman A, Witteman JC (1999) Menopausal status and risk factors for cardiovascular disease. J Intern Med 246(6):521-528. https:// doi.org/10.1046/j.1365-2796.1999.00547.x 
30. Oner P, Mutlu-Turkoglu U, Omer B (1997) Evaluation of the changes in serum lipid profile and ferritin concentrations in relation to body ascorbic acid status in healthy pre- and postmenopausal women. J Nutr Sci Vitaminol (Tokyo) 43(1):1-9. https://doi.org/ 10.3177 jinsv.43.1

31. Wakatsuki A, Sagara Y (1995) Lipoprotein metabolism in postmenopausal and oophorectomized women. Obstet Gynecol 85(4): 523-528. https://doi.org/10.1016/0029-7844(94)00452-J

32. Wang Q, Ferreira DLS, Nelson SM, Sattar N, Ala-Korpela M, Lawlor DA (2018) Metabolic characterization of menopause: cross-sectional and longitudinal evidence. BMC Med 16(1):17. https://doi.org/10.1186/s12916-018-1008-8

33. de Kat AC, Dam V, Onland-Moret NC, Eijkemans MJ, Broekmans FJ, van der Schouw YT (2017) Unraveling the associations of age and menopause with cardiovascular risk factors in a large population-based study. BMC Med 15:2. https://doi.org/10.1186/ s12916-017-0841-5
34. El Khoudary SR, Hutchins PM, Matthews KA, Brooks MM, Orchard TJ, Ronsein GE, Heinecke JW (2016) Cholesterol efflux capacity and subclasses of HDL particles in healthy women transitioning through menopause. J Clin Endocrinol Metab 101(9):3419-3428. https://doi.org/10.1210/jc.2016-2144

35. Miller VT (1995) Effects of estrogen or estrogen/ progestin regimens on heart disease risk factors in postmenopausal women. Jama 273(3):199. https://doi.org/10.1001/jama.1995.03520270033028

36. Walsh BW, Kuller LH, Wild RA, Paul S, Farmer M, Lawrence JB, Shah AS, Anderson PW (1998) Effects of raloxifene on serum lipids and coagulation factors in healthy postmenopausal women. Jama 279(18):1445-1451

Publisher's note Springer Nature remains neutral with regard to jurisdictional claims in published maps and institutional affiliations. 